\title{
High-Pressure-Induced Planarity of the Molecular Arrangement in Maleic Anhydride
}

\author{
Yuxiang Dai, ${ }^{\dagger}$ Kai Wang, ${ }^{*}{ }^{\dagger}$ Xiaodong $\mathrm{Li}^{\ddagger},{ }^{\ddagger}$ and Bo Zou, ${ }^{*}{ }^{\dagger}$ \\ ${ }^{\dagger}$ State Key Laboratory of Superhard Materials, Jilin University, Changchun 130012, \\ China. \\ ${ }^{\ddagger}$ Beijing Synchrotron Radiation Laboratory, Institute of High Energy Physics, Chinese \\ Academy of Sciences, Beijing 100039, China.
}


TABLE S1 Assignment of the Major Raman Bands of Maleic Anhydride

\begin{tabular}{cc}
\hline Assignment of the Major Raman Bands of Maleic Anhydride before Phase Transitions \\
\hline Frequency $\left(\mathrm{cm}^{-1}\right)$ & Assignment \\
\hline 3179 & $\mathrm{C}-\mathrm{H}$ stretching \\
3116 & $\mathrm{C}-\mathrm{H}$ stretching \\
1873 & $\mathrm{C}=\mathrm{O}$ stretching \\
1846 & $\mathrm{C}=\mathrm{O}$ stretching \\
1775 & $\mathrm{C}=\mathrm{O}$ stretching \\
1747 & $\mathrm{C}=\mathrm{O}$ stretching \\
1617 & $\mathrm{C}=\mathrm{C}$ stretching \\
1590 & $\mathrm{C}=\mathrm{C}$ stretching \\
1563 & $\mathrm{C}-\mathrm{O}$ stretching \\
1530 & $\mathrm{C}-\mathrm{O}$ stretching \\
1306 & $\mathrm{C}-\mathrm{C}$ stretching \\
1304 & $\mathrm{C}-\mathrm{O}$ stretching \\
1278 & $\mathrm{C}-\mathrm{O}$ stretching \\
1261 & $\mathrm{C}-\mathrm{O}$ stretching \\
1234 & $\mathrm{C}-\mathrm{O}$ stretching \\
1065 & $\mathrm{C}-\mathrm{O}$ stretching \\
1057 & $\mathrm{C}-\mathrm{H}$ bending \\
950 & $\mathrm{C}-\mathrm{C}$ stretching \\
890 & $\mathrm{C}-\mathrm{O}$ stretching \\
863 & $\mathrm{C}-\mathrm{C}$ stretching \\
762 & $\mathrm{C}-\mathrm{H}$ bending \\
700 & ring deformation \\
638 & ring deformation \\
632 & ring bending \\
556 & $\mathrm{C}=\mathrm{O}$ bending \\
548 & ring deformation \\
537 & ring deformation \\
406 & ring deformation \\
340 & ring bending \\
269 & $\mathrm{C}=\mathrm{O}$ wagging \\
268 & ring bending \\
188 & $\mathrm{C}=\mathrm{O}$ wagging \\
\hline & \\
\hline
\end{tabular}


TABLE S2 Lattice Parameters of Maleic Anhydride at 0.6 GPa

\begin{tabular}{lccccc}
\hline symmetry & pressure (GPa) & $a(\AA)$ & $b(\AA)$ & $c(\AA)$ & $\beta(\operatorname{deg})$ \\
\hline monoclinic & 0.6 & $15.25(7)$ & $4.98(2)$ & $10.14(8)$ & $94.25(0)$ \\
\hline
\end{tabular}

